the law did not meet the benchmark of $95 \%$ recommended by the World Health Organization.

Only $81.3 \%(\phi=7.79, \mathrm{p}<0.01)$ were timely vaccinated for the first time against hepatitis B, only $35.5 \%(\phi=19.92$, $\mathrm{p}<0.01)$ received the third vaccination on time; and only $77.9 \%(\phi=9.25, \mathrm{p}<0.01)$ were vaccinated against tuberculosis. The proportion of children timely vaccinated against whooping cough, diphtheria, tetanus and polio, was $45.5 \%$ $(\phi=19.80 ; \mathrm{p}<0.01)$, and those who have completed the full vaccination set until 6 months accounted for $22.3 \%(\phi=$ 22.42, $\mathrm{p}<0.01$ ).

The coverage of children with vaccination against measles, rubella and mumps in the decreed period (12 months) was also insufficient and represented $42.5 \%(\phi=$ of 17.03 , $\mathrm{p}<0.01)$ which does not guarantee epidemiological welfare of the territory, and in case of introduction of infection it can lead to its spread among the unvaccinated population.

According to the vaccination documentation, parents refusal to vaccinate and medical conditions (prematurity, low weight at birth, respiratory distress, neonatal jaundice, maternal HIV-infection) were causes of failure to immunize children against tuberculosis and hepatitis B in maternity. Temporary medical exemptions to immunization (acute respiratory infection, intestinal infection, allergic dermatitis), delayed appearance to the vaccination, and parents refusal to vaccinate were the leading causes of violation of the timing for subsequent vaccination in the outpatient department.

Thus, the examination of outpatient medical records revealed serious shortcomings in terms of preventive vaccination regulated by National calendar and timing of vaccination in young children, which forms a group of people susceptible to infection which is sufficient for activation of the epidemic process.

\section{4 \\ doi: 10.15789/2220-7619-2018-4-1.4 \\ REGISTRATION OF DYSENTERY SONNEI CASES IN SPECIALIZED MEDICAL INSTITUTIONS OF THE LENINGRAD REGION}

O.A. Istorik ${ }^{1}$, E.A. Mikhailova ${ }^{1}$, M.A. Chorniy ${ }^{1}$, Z.N. Matveeva ${ }^{2}$, A.A. Porin ${ }^{2}$, L.A. Kaftyreva ${ }^{2}$

${ }^{1}$ Department of Rospotrebnadzor of the Leningrad Region,

St. Petersburg, Russia; ${ }^{2}$ St. Petersburg Pasteur Institute,

St. Petersburg, Russia

We conducted an epidemiological investigation of infectious disease group focus that emerged in January 2018 in one of the specialized hospitals in the Leningrad region.

Within eight days in one of hospital departments nine patients and the department nurse developed similar symptoms: abdominal pain, fever and diarrhea. In all cases, "Acute gastroenteritis of mild severity" was initially diagnosed. Subsequently every case had $S$. sonnei positive result of feces examination. In blood sera specific antibodies were detected in diagnostic titres.

The presumed source was the patient admitted to the hospital in the incubation stage. Numerous violations of the hospital sanitary and epidemiological regime as well as personal hygiene by patients and staff made it possible to implement a contact-household transmission route and the emergence of a group illness. Patients were transferred to the clinic of infectious diseases, preventive and anti-epidemic measures in the hospital allowed to quickly stop the outbreak. Strains of $S$. sonnei characterized by identical enzymatic properties and antibiogram. The strains were resistant to ampicillin, tetracyclines, chloramphenicol, sulfonamides, trimethoprim, and were characterized by a low level resistance to fluoroquinolones. Sensitivity persisted in the expanded spectrum of cephalosporins (ceftazidime, cefatoxime, cefepime) and carbapenems (merapenem), aminoglycosides (gentamicin, tobramycin, amikacin), nitrofurans.

The genotyping of isolated strains using RAPD-PCR, like the phenotypic methods, showed their identity.

During laboratory and clinical examination of medical and technical personnel of the department, as well as workers of the food unit, shigelosis patients and carriers of $S$. sonnei were not identified.

A shigellosis outbreak in a specialized hospital with a long stay of patients, caused by $S$. sonnei, arose as a result of the introduction of infection to the department, and had nosocomial spreading through a contact-household transmission route.

1.5 doi: 10.15789/2220-7619-2018-4-1.5

\section{ZIKA FEVER IN THE WORLD AND THE RISK OF ITS DISTRIBUTION IN THE RUSSIAN FEDERATION}

\section{A.B. Khaitovich, M.E. Lukyanova}

V.I. Vernadsky Crimean Federal University, Simferopol, Russia

Zika fever is an arbovirus transmissible disease caused by Zika virus and transmitted by mosquitoes Aedes aegypti and Aedes albopictus. Discovered for the first time in 1947 in Uganda, the Zika virus led to diseases among people in the 1960s-1980s in Africa and Asia, then to outbreaks in 2007 on the island of Yap in the Western Pacific and in 2013-2014 in French Polynesia. The epidemic, which began in the fall of 2015 in South and Central America, reached 70 countries and led to more than half a million cases. The aim and objectives of the work are to analyze the dynamics of the incidence of Zika fever and to identify tendencies in the distribution in the regions of the world, to identify the features of mosquito biology and to assess the factors affecting the spread of these diseases and the incidence among humans, and to determine the risks of spreading the fever to other areas.

With the help of GIS, an epidemiological analysis of the incidence and spread of Zika fever was carried out, and the features of mosquito biology were determined. Information about the incidence is obtained from the information messages of the WHO and Rospotrebnadzor Statistical and graphical methods of investigation were used to process the results.

The epidemic process of Zika fever in South and Central America has come to an end. The analysis of morbidity, which was carried out, showed that the outbreak was uneven in time and space, with 6 phases of the epidemic process identified. The obtained data for biological characteristics of populations of two species of mosquitoes allowed us to attribute Aedes aegypti to the group of more important species for epidemic spread - to the main carriers, and Aedes albopictus to less significant, secondary carriers. The information obtained does not give an accurate prediction of the further spread of Zika fever in different regions. But it can be assumed that there can be a region in the Russian Federation - the Black Sea coast of the Russian Federation (the Caucasus and the Crimea), which should be assessed at present as unfavorable for the formation of a focal point for augmented transmission of infection. However, with changing climatic conditions, the situation may change. The causative agents of some fevers may expand the regions of distribution and this is associated with the same types of mosquito vectors as in the case of Zika fever. 
The expansion of the geography of mosquito vector regions and their adaptation to colder and drier climatic conditions with the potential for transmission of infection gradually expand the area and require scientific study and monitoring of hemorrhagic fever viruses and Zika virus in particular.

\section{6 doi: 10.15789/2220-7619-2018-4-1.6 MODERN PROBLEMS OF INFECTIOUS DISEASES PREVENTION IN PUBLIC HEALTH OF REPUBLIC OF GUINEA}

O.K. Konstantinov ${ }^{1}$, M.Y. Boiro ${ }^{1}$, N.E. Lama ${ }^{2}$, E.V. Verigina ${ }^{3}$ ${ }^{1}$ Research Institute of Applied Biology of Guinea, Kindia, Republic of Guinea; ${ }^{2}$ Ministry of Health and Public Hygiene, Conakry, Republic of Guinea; ${ }^{3}$ Central Research Institute of Epidemiology, Moscow, Russia

Republic of Guinea is one of the developing countries of West Africa. Of the whole GDP only 400-500 USD are on one habitant annually. More than half habitants live down the poverty level. The country carries a heavy burden of many infectious diseases.

The aim of the study was to describe the actual problems of infectious diseases prevention in public health of Republic of Guinea.

Statistical data from public health institutions of the country were used. Methods - descriptive and analytical

Population size of Guinea increases and numbers now more than 12 millions habitants. The public health structure of the country includes: 925 first-aid posts, 410 prefecture and regional centers of health, 8 communal health centers, 26 prefecture, 7 regional and 3 national hospitals. Though medical aid is below of the regional norms of WHO. In Guinea 74 nosoforms of infectious and non infectious diseases are registered. The part of infectious diseases in the morbidity structure was 38-44\% (1.9-2.5 millions cases annually). The most widespread infections were malaria, acute respiratory (ARI) and intestinal infections. In the mortality structure ARI occupied $12.5 \%$, malaria $-10 \%$, acute intestinal infections $6 \%$, HIV infection consists $5 \%$. The sexual transmissible infections are widespread: 200 thousand cases in a year. The outbreaks of measles, meningitis, cholera, Yellow Jack are registered too. Prevention measures are actively conducted. All little children are vaccinated obligatory against: poliomyelitis (4 ${ }^{\text {th }}$ time), BCG, kombi (diphtheritic, tetanus and pertussis), measles, Yellow Jack. The pregnant women receive anti malaria drugs as prevention and anti tetanus vaccine. The HIV positive pregnant women receive antiretroviral therapy. One realizes health education programs, particulary among the youth. One popularizes the use of contraceptives, organizes centers of family planning. However this activity meets some difficulties because of the low education level, some religious and socio-cultural customs of population, what was visually revealed during Ebola fever outbreak. The vaccination program meets such difficulties as cold chain, lack of qualified medical personnel, lack of medicaments and technical equipment of diagnostics laboratories, which number is insufficient. In order to dissolve these problems of diagnostics, control and prevention of infectious diseases in 2018 the 3 -years program "National strategy on medical biology" was elaborated.

In spite of difficulties the public health of Guinea goes on to develop. The realization of the "National strategy" will help in strengthening of health and welfare of population of Guinea.
1.7

doi: 10.15789/2220-7619-2018-4-1.7

\section{FECAL-ORAL MECHANISM IN THE GROUP AND EPIDEMIC STRUCTURES ON THE TERRITORY OF THE ROSTOV REGION WITHIN 10 YEARS}

E.V. Kovalev, E.G. Erganova, S.A. Nenadskaya, S.S. Slis', G.A. Miroshnichenko

Rostov Region Surveillance Directory, Rostov-on-Don, Russia

Infections with the fecal-oral transmission take one of the leading places in the infection pathology of population including economically developed counties, going only after respiratory infections among mass infectious diseases.

In Rostov region according to the form of statistic observation No. 23-17 "Information about outbreaks of infectious diseases", within 10 years from 2008 until 2017 th 104 cases of group and outbreak morbidity was reported, with 1853 caseload, including 1130 children under 17 years (61.0\% of the total number of cases).

In structure the main gravity in the region lies on the fecal-oral mechanism (76.9\%) and also on aspiration $(10.6 \%)$ and others $(12.5 \%)$.

The spread was occurred by food - in 47 cases ( $45.2 \%)$, by water - in $14(13.5 \%)$, by household contacts - in 19 (18.3\%), airborne-in 11 (10.5\%) and other - 13 (12.5\%)

Etiological factors in group and outbreak morbidity were bacterial pathogens of infection: Salmonella Enteritidis (11), Salmonella Typhimurium (3), Salmonella Seegefeld (1), Salmonella Muenchen (1), Salmonella Isangi (1), Salmonella spp. (1), Shigella sonnei (6), opportunistic pathogenic microflora (7) (Staphylococcus aureus, Proteus vulgaris, Citrobacter, Escherichia coli, Enterobacter aerogenes), viral aetiology: group A rotaviruses (15), 2 genotype Noroviruses (18), hepatitis a virus (9), Enteroviruses (14), mixed etiology (1), undetermined etiology (4), measles virus (5), Crimean-Congo hemorrhagic fever (1), parvovirus in 19 (1), influenza (1), tuberculosis (1), chickenpox (1), community-acquired pneumonia (1) and epidparotite (1).

Improvement of decoding of acute intestinal infections (ОКИ) and further development of laboratory diagnostics in etiological structure dramatically changes were noticed: the increasing epidemiological importance is given to "intestinal" viruses, the intensity of circulation of which has increased in recent years (enteroviruses, rotaviruses and noroviruses).

The changed structure of the epidemic process including acute intestinal infections, the growth of viral infections requires new approaches in the improvement of activities and diagnosis, anti-epidemic supply and prevention activity.

1.8 doi: 10.15789/2220-7619-2018-4-1.8

THE CHARACTER OF MICROBIOTA IN THE INTENSIVE CARE UNIT OF THE CHILDREN'S HOSPITAL AND ITS EPIDEMIOLOGICAL SIGNIFICANCE

L.A. Kraeva ${ }^{1,2}$, L.B. Vainer, K.E. Vinogradova

${ }^{1}$ St. Petersburg Pasteur Institute, St. Petersburg, Russia;

${ }^{2}$ S.M. Kirov Military Medical Academy, St. Petersburg,

Russia; ${ }^{3}$ Pavlov First St. Petersburg State Medical University, St. Petersburg, Russia

As is known, the risk of infections associated with medical care is highest in intensive care units, especially in the department of pathology of newborns and premature infants. The aim of the study was to study the composition and characteristics of bacteria circulating in the intensive care unit of the children's hospital. 